\title{
Gobernar(SE) EN NOMBre DE LA CUltura. Interculturalidad y educación para grupos étnicos en Colombia
}

\author{
AXEL RoJAS \\ UNIVERSIDAD DEL CAUCA \\ axelrojasm@gmail.com
}

Resumen

[l objetivo de este artículo es mostrar el despliegue de la interculturalidad en ColomLbia, particularmente el proceso en el cual se produce su articulación con programas de educación para grupos étnicos. Basado en la noción de gubernamentalidad, argumentaré que, más que como una alternativa al multiculturalismo, la interculturalidad opera como una de sus tecnologías de gobierno de la alteridad. Es decir, como un conjunto de dispositivos encaminados a la orientación de la conducta de ciertas poblaciones e individuos, en este caso en nombre de su diferencia cultural. PALABRAS CLAVE: multiculturalismo, interculturalidad, educación para grupos étnicos, gubernamentalidad.

\section{(Self)-Government in the Name of Culture: \\ INTERCULTURALITY AND ETHNIC \\ Group Education in Colombia}

\begin{abstract}
The purpose of this article is to trace the rise of interculturality in Colombia by focusing on the process by which it has become articulated to education programs aimed at ethnic groups. Working from the concept of governmentality, I will argue that instead of being an alternative to multiculturalism, interculturality rather operates as one of its technologies of governing alterity. So to speak, it operates as a series of devices aimed at the orientation of the conduct of certain populations and individuals, in this case, on behalf of their cultural difference.
\end{abstract}

KEY WORDS: multiculturalism, interculturalism, ethno-education, governmentality. 


\section{INTRODUCCIÓN ${ }^{1}$}

Cobre la cultura recae hoy en día una atención inusual, que se expresa tanto en las formas más aceptadas y extendidas de pensar el momento histórico como en las características de muchas de sus disputas; podríamos decir, con Trouillot, que "ahora la cultura explica todo" (2OII, I76). Como parte de esta visibilización creciente de la cultura —o las culturas- han surgido novedosos problemas:

I El presente artículo es resultado de una investigación realizada para optar al título de magíster en Estudios Culturales de la Universidad Javeriana. Una parte de los argumentos presentados ahora se encuentran en la tesis elaborada con tal propósito. nuevos objetos de atención e intervención política y académica. Uno de los más destacados en el momento es la cuestión multicultural, la creciente certeza de habitar un mundo poblado por distintos grupos culturales; y junto a este, el problema de las relaciones entre dichos grupos (relaciones interculturales). Se trata de un problema que ha llegado a dispersarse de tal forma que abarca hoy en día ámbitos cada vez más amplios de la vida social, como la salud, el derecho, la economía y la filosofía, para mencionar solo algunos.

Con el fin de comprender la manera en que las relaciones entre culturas han adquirido tal relieve en las preocupaciones de nuestro tiempo, analizaré los programas de educación indígena y etnoeducación en Colombia. Me centraré aquí en el problema de las relaciones entre culturas, que en Colombia tuvo su emergencia en la primera mitad del siglo xx, en el marco de las políticas indigenistas promovidas por organismos como la Organización de las Naciones Unidas para la Educación, la Ciencia y la Cultura (Unesco), agencias de cooperación internacional en asocio con entidades estatales y, con frecuencia, organizaciones sociales. Estos proyectos tuvieron un primer momento de institucionalización en la década de los cuarenta, luego de la realización del Primer Congreso Indigenista Interamericano (I94I) en Pátzcuaro (Ballesteros y Ulloa 196I); en ese primer momento, la interculturalidad era empleada como una categoría descriptiva, que hacía referencia a los espacios y relaciones de contacto entre poblaciones indígenas y poblaciones mestizas (Aguirre Beltrán 1957). Progresivamente, el concepto de interculturalidad se irá haciendo más prescriptivo, y entonces las trayectorias de la educación indígena y de la etnoeducación adquieren especial relevancia 
para comprender el despliegue de programas y proyectos que se proponen como alternativas para modificar las relaciones históricas de subordinación entre grupos que conviven en espacios multiculturales.

Con el paso del tiempo, la interculturalidad comenzará a ser conceptualizada como proyecto; no como algo existente, sino como algo por alcanzar. Los programas que empiezan a ser calificados como interculturales (educación intercultural, epidemiología intercultural, filosofía intercultural, etc.), o sustentados en principios entre los que se incluye la interculturalidad, son presentados como proyectos dirigidos a la transformación de las formas históricas de sometimiento de poblaciones y de imposición de saberes, de tal manera que las relaciones jerarquizadas sean remplazadas por otras de tipo horizontal. Simultáneamente, a medida que se consolida esta enunciación de la interculturalidad como proyecto, se produce un nuevo giro en los debates académicos, que la empiezan a plantear como una alternativa al multiculturalismo.

En tal sentido, en este artículo me propongo mostrar cómo, más que una alternativa al multiculturalismo, la interculturalidad opera como tecnología de gobierno de la alteridad: como un programa que define la manera en que deberán comportarse quienes son pensados como los otros de la nación. Con este propósito, analizo las políticas de educación para indígenas y afrodescendientes que han sido promovidas por el Estado, organismos multilaterales, sectores de la academia y organizaciones sociales, hace ya casi un siglo. Una de las características de estas políticas es haber logrado presentarse como resultado de una aspiración ancestral y como componente esencial de las políticas étnicas. Sin embargo, mostraré cómo emergen en las entrañas de la burocracia estatal —nacional y transnacional—, ligadas a las políticas de la academia y en un momento histórico particular. En esta perspectiva, tomo distancia de posturas más comunes en Colombia y América Latina, que han entendido la interculturalidad como parte de un proyecto político y epistémico que recogería la voz o los principios ideológicos de organizaciones sociales, indígenas y afrodescendientes (Bolaños et ál. 2004; Rappaport 2008; Walsh 2004, 2009); es decir, aquellas que la entienden como un proyecto alternativo agenciado por los grupos étnicos de acuerdo con sus intereses, y que desconocen su orígenes y trayectorias. 
Ahora bien, elaborar una crítica del multiculturalismo no supone desconocer su potencial democratizante. Es indudable que pensar en términos culturalistas ha posibilitado poner en cuestión los proyectos imperiales de subalternización de poblaciones y saberes, lo cual ha potenciado novedosas expresiones de la política. Al hacer énfasis en los riesgos y contradicciones de proyectos como el de la interculturalidad, lo que se intenta es proponer nuevos elementos para la discusión y hacer visibles algunos procesos en los que se produce la sedimentación del culturalismo, que es el nuevo sentido común de la época en que vivimos. Un sentido común que clausura amplias posibilidades de lo político, incluso cuando promueve la idea de estar ampliando sus horizontes.

\section{El multiculturalismo como \\ GUBERNAMENTALIZACIÓN DE LA CULTURA}

De

e manera semejante a como durante los siglos XIX y xx se produjo un proceso de gubernamentalización, que consistió en la progresiva multiplicación de las formas de gobierno de los seres humanos y en la creciente capacidad del Estado para gobernar y ser demandado como aparato de gobierno (Foucault 2006), durante las décadas finales del siglo xx y lo que va corrido del presente se ha producido un proceso ascendente de gubernamentalización de la cultura. La noción de gubernamentalidad permite ver cómo operan las estrategias, tácticas y autoridades que se plantean como propósito el bienestar de individuos y poblaciones, o la eliminación de los conflictos que los afectan, y que en consecuencia procuran conducirlos a la adopción de mejores prácticas de vida o, en general, a la alteración de su conducta (Inda 2008). Desde esta perspectiva podemos analizar con mayor complejidad los entramados de relaciones de saber-poder en los que individuos y poblaciones llegan a orientar sus acciones de acuerdo con ciertos principios y conceptos cuya autoridad aparece como incuestionable, y podemos también rastrear la manera en que dichos conceptos se enraízan en prácticas y se hacen objeto de programación de la conducta de estas poblaciones, tanto por otros como por sí mismas. Es decir, comprenderemos a la vez cómo son gobernadas ciertas poblaciones y cómo se gobiernan 
a sí mismas, y que ciertos proyectos que fueron concebidos inicialmente como técnicas de integración son reclamados hoy como alternativas y expresiones de resistencia.

Según plantea Foucault, la gubernamentalidad está relacionada con un tipo de poder que tiene como blanco a la población ${ }^{2}$; se trata de la preeminencia de un tipo particular de poder que puede ser llamado gobierno, y de un proceso mediante el cual el Estado ha sido progresivamente gubernamentalizado (Foucault 2006, I36). Así, resulta indispensable comprender que el gobierno no es algo que el Estado despliega sobre los ciudadanos, sino una forma de poder ejercida por diversas entidades, organizaciones

2 La noción de población es capital en el trabajo de Foucault, quien la desarrolló, entre otras, en las clases del Collège de France del mes de enero de 1978 (2006, 15-IO8).

3 Valga mencionar que nociones como diversidad cultural o incluso multiculturalidad operan como formas de nombrar la alteridad: refieren a la existencia de grupos humanos que ocupan un e individuos, a quienes se les reconoce la autoridad para intervenir sobre la conducta de los seres humanos. El gobierno es un tipo de poder ejercido incluso por aquellos que en apariencia son ajenos a él, como pueden ser los maestros, los indígenas, los afrodescendientes, los activistas y los académicos, entre otros. Su blanco principal son las poblaciones, lo cual implica que las autoridades políticas y de otra índole buscan intervenir sobre aspectos específicos de la conducta humana con el propósito de mejorar su seguridad, longevidad, salud, prosperidad y felicidad (Inda 2008, 6).

Así pues, cuando hablo de gubernamentalización de la cultura me refiero al proceso en que unas formas particulares de gobierno de individuos y poblaciones han llegado a estar sustentadas en razones culturalistas, es decir, a formas de gobierno que se caracterizan y se sustentan en la proliferación de saberes expertos que describen, analizan y prescriben las formas adecuadas de comprensión y conducción de las sociedades que son concebidas y se conciben como multiculturales. Este proceso está relacionado con la progresiva certeza de nuestro tiempo de habitar un mundo compuesto por grupos culturales, en el que las formas de entender lo social están marcadas por nociones como cultura y diversidad cultural, por saberes como la antropología y el derecho, y por la presunción de que ciertas expresiones de la política y ciertos sujetos tienen mayores posibilidades y legitimidad en sus intervenciones para transformar el mundo ${ }^{3}$. A este proceso, que llamo gubernamentalización de la cultura, lo conocemos 
lugar de otredad en relación con aquel o aquellos grupos que ocupan el lugar de mismidad, en un momento determinado, en una formación social específica. Es así como, en los países históricamente sometidos a la colonización hispana, la multiculturalidad ha venido siendo entendida como sinónimo de plurietnicidad; y la etnicidad, como equivalente a la presencia de poblaciones sometidas en el marco de la experiencia colonial (indígenas y afrodescendientes, específicamente), mientras que en países como Estados Unidos y algunos de Europa, la etnicidad se refiere a la presencia de poblaciones de inmigrantes. Es decir, la multiculturalidad no es un hecho objetivo, siempre igual en todos los momentos y lugares, sino que adquiere expresiones particulares según los contextos.

4 Para un análisis más completo de estas problemáticas, resultaría interesante estudiar también cómo la interculturalidad fue progresivamente incorporada en las demandas políticas de organizaciones sociales, en las conceptualizaciones de los intelectuales indígenas y afrodescendientes y en los proyectos curriculares realizados por ellos mismos. Aunque esto sería objeto de otro texto.

5 Jonathan Inda, basado en el trabajo de Foucault, propone una analítica de lo moderno en la que considera tres dimensiones del gobierno. "En primera instancia están las razones de como multiculturalismo: un proyecto de gobierno de individuos y poblaciones sustentado en razones culturales. Junto a él, y como uno de sus elementos constitutivos, emerge el problema de las relaciones entre culturas o interculturalidad, la cual es una tecnología del multiculturalismo y por eso se la encuentra comúnmente ligada a ciertos proyectos como una razón política.

Con el objetivo de analizar históricamente cómo ha operado el multiculturalismo en Colombia, resulta especialmente esclarecedor estudiar la manera en que las relaciones entre culturas han sido objeto de atención e intervención de las políticas de Estado $^{4}$. Desde inicios del siglo xx, es posible observar cómo la interculturalidad se encuentra ligada a programas o proyectos dirigidos a las poblaciones que son pensadas como los otros de los proyectos nacionales a lo largo y ancho del planeta (Montalto I978; Redden y Ryan I95I; Walsh 1973). La interculturalidad opera como objeto de atención académica y como principio o meta de programas políticos, educativos, epidemiológicos, administrativos o filosóficos, referidos a poblaciones indígenas, afrodescendientes y migrantes, principalmente. Para comprender cómo ha sucedido este proceso, es necesario analizar la manera en que la cultura ha llegado a ser un argumento en nombre del cual se busca conducir la conducta de individuos y poblaciones: es decir, el proceso mediante el cual se constituye en razón de gobierno. De otro lado, es necesario analizar las tecnologías, que son la forma pragmática que adquieren dichas razones (Inda 2008), y que se materializan en documentos, dispositivos, declaraciones, políticas y proyectos, en los que se expresan las voces de instituciones y expertos autorizados para definir y prescribir las conductas requeridas ${ }^{5}$. 
En este sentido, analizo cómo opera la interculturalidad entendida como tecnología, a través de un proyecto concreto, que es el de la etnoeducación; un proyecto que define quiénes son considerados otros, en qué circunstancias, de acuerdo con qué atributos; $y$ al mismo tiempo, cómo deben relacionarse con la cultura, los conocimientos y las políticas en relación con los cuales son considerados otros $^{6}$. Aunque no es la única tecnología del multiculturalismo, sus trayectorias, objetivos, conceptualizaciones e institucionalidades, así como las poblaciones que son objeto de su accionar, permiten comprender cómo opera el proceso de gubernamentalización de la cultura.

\section{EL DESPLiegue DE UNA TECNOLOGÍA}

partir de la década de i980, la
preocupación por las relacio-
nes entre culturas comenzó a gobierno; esta dimensión reúne todas aquellas formas de conocimiento, de experticia y de cálculo que hacen posible que pensemos a los seres humanos como susceptibles de programación política. En segundo lugar están las técnicas de gobierno; al dominio de lo técnico pertenecen los mecanismos prácticos, los instrumentos y los programas por medio de los cuales autoridades de distintos tipos buscan dar forma e instrumentalizar la conducta humana. Finalmente están los sujetos de gobierno; esta dimensión cubre los diversos tipos de identidad individual y colectiva que emergen a partir de, y al mismo tiempo sustentan, la actividad gubernamental" $(2008,2)$.

6 El interés por la cultura en este artículo no tiene que ver con la validez o exactitud de una definición empleada por quienes hablan de la interculturalidad, o cualesquiera otros; lo que me interesa es ver cómo ella opera como principio de inteligibilidad de lo social y cómo se produce su inserción en entramados de saber-poder. Cuando me refiero a la palabra cultura, lo que me interesa es cómo en su nombre se diseñan y ejecutan programas de gobierno de poblaciones; programas que pueden ser y son con frecuencia requeridos y dirigidos por aquellos grupos objeto de gobierno. Es decir, mi objetivo es comprender cómo individuos y poblaciones son gobernados y se gobiernan a sí mismos en nombre de la cultura.

7 En Colombia, grupos étnicos es una categoría de uso social y académico que hace referencia a poblaciones indígenas, afrodescendientes y gitanas. Aunque de manera bastante distinta en cada caso, estos tres grupos encarnan de manera predominante lo que se entiende como diferencia cultural. políticas y académicas promovidas por grupos étnicos ${ }^{7} \mathrm{o}$ en nombre de ellos. Las condiciones que hicieron posible la emergencia y despliegue de la interculturalidad tuvieron que ver, inicialmente, con el indigenismo - ligado a la institucionalización de políticas de Estado y de programas académicos de Antropología, principalmente-, así como con la globalización de los derechos humanos y los derechos culturales en el periodo de posguerra, y con las críticas al colonialismo (Anaya 2005; Díaz-Polanco et ál. 1979/1987). No menos importantes fueron la presencia de intelectuales que reclamaron su autoridad para hablar por (o desde) estas poblaciones y sus intereses; y la definición, desde 
el Estado, de mecanismos oficiales de interlocución, de las formas aceptadas de reconocimiento y de los criterios que al final establecerían cuáles voces serían escuchadas al hablar por la diferencia. A la par de estos procesos de tipo institucional se dio la internacionalización de redes de organizaciones sociales, que constituyeron múltiples formas de intercambio y apoyo mutuo, además de lenguajes y perspectivas compartidas para expresar sus demandas y plantear vías de solución (Bonfil 1979; Cunin en prensa; Escobar 2010, 289-298; Santamaría 2008; Wade 2007). Esta conjunción de factores contribuyó a dar forma a proyectos en los que la interculturalidad aparece como un fin asociado a la transformación de las relaciones históricas entre grupos humanos con culturas diferentes.

Un ejemplo de ello son los programas de etnoeducación que, sustentados en saberes como los de la antropología, la lingüística y el derecho, definen los rasgos que deben caracterizar la educación que reciban o adelanten por sí mismos los indígenas y los afrodescendientes; un rasgo de esta tecnología particular es la centralidad que adquiere en ella la noción de cultura. Como veremos a continuación, los proyectos de etnoeducación son también un mecanismo de difusión de la interculturalidad, a la que se enuncia como un principio en nombre del cual se realizan los programas de educación para grupos étnicos.

Hoy en día es común que la etnoeducación sea reclamada o presentada como un derecho étnico, tanto por organizaciones sociales como por académicos y funcionarios. Ello pareciera significar que este tipo de proyectos educativos son esenciales para la preservación de la vida de aquellas poblaciones que se reconocen y son reconocidas como grupos étnicos; de hecho, es común que se argumente que este derecho es fundamental para la preservación de sus culturas. Pero, ¿cómo es que un derecho tan importante solamente llegó a adquirir tal importancia en las últimas décadas? Si analizamos un poco la historia, veremos que la preocupación por la educación de los indígenas y los afrodescendientes no es nueva, aunque comúnmente fue pensada como mecanismo de nivelación de estos grupos a los estándares de civilización del momento. Es decir, no se trató de la preservación de sus culturas, sino más bien de su borramiento y de la adecuación de su comportamiento a formas de ver el mundo propias de las élites de cada época. Vale preguntarse entonces, 
¿hasta dónde estas nuevas educaciones son distintas a las anteriores? ¿La etnoeducación y la interculturalidad son alternativas o inherentes al nuevo proyecto civilizatorio?

Desde mi punto de vista, la interculturalidad y la etnoeducación solo son posibles en el actual momento histórico, y son una de sus más depuradas expresiones, muestran la manera concreta como se materializa el multiculturalismo en Colombia.

En I982, el Ministerio de Educación Nacional de Colombia dio un cambio a su política de educación indígena, que a partir de entonces sería conocida bajo la conceptualización de etnoeducación. Según la coordinadora del programa en ese momento, a partir de entonces " [...] empiezan a generarse a lo largo y ancho del país, experiencias educativas encaminadas a la elaboración de programas bilingües-interculturales" (Bodnar 1986, ii). Unos años después, en octubre de 1986, la política de etnoeducación sería caracterizada con tres rasgos fundamentales: ser participativa, bilingüe e intercultural (94). Estos tres rasgos expresan claramente el sentido de la política; los dos primeros eran una muestra de la pervivencia de las políticas indigenistas de integración iniciadas a finales de la primera mitad del siglo xx: la participación buscaba que el indígena aprendiera a gobernarse a sí mismo, y el bilingüismo, entendido como mecanismo de castellanización, apuntaba a que los indígenas aprendieran el idioma nacional ${ }^{8}$. Aunque para los ochenta las políticas indigenistas estaban en proceso de transformación y desde diferentes frentes se había llamado la atención acerca de su carácter homogeneizan8 La lingüística es uno de los saberes académicos que constantemente estarán presentes en el trabajo educativo dirigido a poblaciones indígenas, en parte debido al peso que se les otorgó a las lenguas indígenas en la transmisión y reproducción de la cultura, un atributo que se tornaría infaltable en los discursos de sí y de la diferencia, producidos en nombre de los grupos indígenas $\mathrm{y}$ - en menor medida, de los afrodescendientes - vinculados a este tipo de iniciativas.

te, también es cierto que para entonces dichas políticas habían conseguido un alto grado de institucionalización, e incluso de legitimidad y demanda social. En otras palabras, aunque se cuestionaba al indigenismo, en las nuevas políticas permanecían muchas de sus lógicas y de sus técnicas de intervención.

Lo que podría considerarse más novedoso en las políticas de etnoeducación es la presencia de dos aspectos ausentes de los proyectos de educación indígena anteriores. Por un lado, el planteamiento del problema en términos políticos, asociado a 
9 En diciembre de I98I, en una reunión de expertos realizada en Costa Rica, se definía el etnodesarrollo en los siguientes términos: "Entendemos por etnodesarrollo la ampliación y consolidación de los ámbitos de cultura propia, mediante el fortalecimiento de la capacidad autónoma de decisión de una sociedad culturalmente diferenciada para guiar su propio desarrollo y el ejercicio de la autodeterminación, cualquiera que sea el nivel que considere, e implican una organización equitativa y propia del poder. Esto significa que el grupo étnico es [una] unidad político-administrativa con autoridad sobre su propio territorio y capacidad de decisión en los ámbitos que constituyen su proyecto de desarrollo dentro de un proceso de creciente autonomía y autogestión" (Organización de las Naciones Unidas para la Educación, la Ciencia y la Cultura [Unesco] y Facultad Latinoamericana de Ciencias Sociales [Flacso] I98I). La conceptualización más elaborada de etnodesarrollo se encuentra en Bonfil (I982, I3I-I45). la formación de sujetos para la construcción de autonomía, que resultaba del vínculo conceptual con la noción de etnodesarrollo, propuesta en el Grupo de Barbados. Así lo menciona la misma autora: “[...] el diseño de la política y del marco conceptual para la educación indígena expresada en los 'Lineamientos', se enmarcan dentro del concepto de etnodesarrollo, teoría elaborada por el profesor mexicano Guillermo Bonfil Batalla [...]” (Bodnar I986, 92, énfasis del original) ${ }^{9}$. El segundo aspecto novedoso en las políticas de etnoeducación fue la

inclusión de la interculturalidad como una de sus características y horizontes, que significaba plantear la dimensión política del conocimiento, también en concordancia con el proyecto del etnodesarrollo. En este primer momento, la interculturalidad aparece ligada a la capacidad del grupo para tomar decisiones de manera reflexiva; se entiende que "la interculturalidad permite apropiarse (o rechazar) de manera reflexiva y crítica, aquellos elementos de otras culturas que constituyan [sic] al mejoramiento de las condiciones de vida de una población” (Bodnar 1986, 95).

Aunque este es el momento de emergencia de la etnoeducación, la educación para indígenas era objeto de preocupación desde tiempo atrás, pero en términos bastante distintos. El indigenismo interamericano había avanzado significativamente en la institucionalización de programas de aculturación orientados a la integración de los indígenas a la nación, de los que hacían parte los proyectos de educación indígena (Ballesteros y Ulloa I96r). Estas políticas se implementaron en los diferentes países de la región con distintos niveles de institucionalización a lo largo de varias décadas. Fue así que en 1972 se realizó en Bogotá la Primera Reunión de Trabajo sobre Educación Bilingüe en los Grupos Indígenas, convocada por la Dirección General de Integración y Desarrollo de la Comunidad, del Ministerio de Gobierno, uno de cuyos objetivos era: "Difundir y facilitar el análisis de la filosofía y técnica de la utilización de las lenguas 
vernáculas como medio de aprendizaje del castellano entre los grupos nativos para lograr al máximo el desarrollo social, cultural y económico de las comunidades indígenas" (Colombia, Ministerio de Gobierno 1972, 4). Como puede verse, la orientación de estos programas correspondía al talante del indigenismo que predominó hasta los años setenta; desde el propio nombre de la oficina encargada: Dirección de Integración y Desarrollo, hasta el planteamiento explícito de emplear el castellano como vía de integración, haciendo uso para ello de las lenguas vernáculas en un proceso de transición; en otras palabras, se trataba de un espacio para el diseño de una tecnología específica dirigida a la integración de los indígenas.

Sin embargo, la educación indígena y bilingüe, al igual que las discusiones, los proyectos y las experiencias de alfabetización ligadas al trabajo de investigación lingüística no nacieron en este momento, ni como resultado de una política estatal colombiana; ni mucho menos como un proyecto emergido de las organizaciones indígenas. A manera de ejemplo podemos referir cómo, en el mismo año, la revista Cultura Nariñense publicó una entrevista realizada por fray Javier Montoya a un "profesor indígena bilingüe" acerca de su labor en Sibundoy (Putumayo), entre los kamsás (Montoya 1972), en la cual se muestra que para entonces la noción de educación indígena y bilingüe ya era empleada en la relación entre indígenas y misioneros. De hecho, es posible encontrarla mucho antes. En I94I, en el "Acta final del Primer Congreso Indigenista Interamericano", en el punto VI, se proponía: "Que los especialistas y Gobiernos trabajen por el perfeccionamiento y la uniformidad de los métodos y las normas de investigación y educación" (en Ballesteros y Ulloa 196I, 28I). Posteriormente se la encontrará en las memorias de congresos indigenistas (cf. Instituto Interamericano Indigenista 1968, 85-I25), en diversos números de la revista América Indígena (Arana 1976; Bairon 1952; Nahmad 1982; Paulston 1970; Vargas y Loewen 1963) y en algunos balances sobre el indigenismo (Marroquín 1972, I05IIO; Rubio 1957, 39, 66).

Como podemos ver, la aparición de una política de etnoeducación a comienzos de los ochenta no es del todo novedosa. A pesar de que el nombre pueda evocar hoy en día una idea más amplia que la de educación indígena, no fue así en su nacimiento. De hecho, la etnoeducación fue sinónimo de educación 
indígena, al menos hasta mediados de los noventa. Sin embargo, la etnoeducación sí es un hito importante en relación con la problematización de las relaciones entre culturas, y a partir de mediados de los ochenta se constituye en herramienta clave para la difusión de un nuevo sentido de la interculturalidad. Con la etnoeducación la interculturalidad deja de ser una categoría descriptiva empleada para nombrar espacios y relaciones de contacto entre indígenas y otras poblaciones, y se constituye en principio orientador de los proyectos educativos de los indígenas y para ellos: es decir, la interculturalidad da un giro y se hace una categoría prescriptiva.

\section{INSTITUCIONALIZACIONES, CONCEPTUALIZACIONES, APROPIACIONES}

un cuando la educación haya estado ligada históricamente a
proyectos que hoy pueden considerarse como opresores, también
es cierto que en diferentes momentos lo ha estado a proyectos
liberadores o democratizantes. Durante los años setenta y ochen-
ta la educación fue objeto de amplios debates y de propuestas
vinculadas a proyectos de transformación social que alcanzaron
gran fuerza en América Latina y tuvieron repercusión en otras
latitudes (Freire 20oo; Torres I986; Giroux 20o4); la educación
popular es solo una de las muestras más visibles y de mayor in-
fluencia. Ligada a proyectos escolarizados y a otros que tuvieron
su lugar fuera de las aulas, incidió con fuerza en la aparición
de experiencias educativas locales, gran parte de ellas ligadas
a procesos de organización social. Para finales de los setenta y
durante la década de los ochenta, estos proyectos eran el foco
de discusión acerca de problemáticas locales diversas, como el
acceso a servicios públicos, las estructuras de tenencia de la tierra
o las historias locales y las trayectorias políticas de los sectores
populares (Bonilla et ál. I972; Fals Borda I985).
Cuando la etnoeducación surgió en el escenario nacional,
diversos sectores indígenas, organizaciones sociales y grupos de
maestros de comunidades negras contaban con experiencias en el
campo educativo, en muchos casos vinculadas a sus particulares
proyectos políticos. Por ejemplo, mientras organizaciones como
el Movimiento Cimarrón luchaban contra el racismo hacia los 
negros y denunciaban el papel de la educación en su reproducción (Mosquera 1986), otras, como el Consejo Regional Indígena del Cauca (CRIC), crearon sus programas de educación y avanzaron en la formación de maestros, en el diseño de materiales y en la gestión de proyectos educativos (Bolaños et ál. 2004). De otra parte, este fue un momento clave para la conceptualización de la educación como derecho, algo que tendría especial eco en las demandas indígenas y en la forma en que se concebirían las nuevas políticas de educación indígena.

A comienzos de los ochenta, empezó el proceso de institucionalización de la etnoeducación como política estatal, inicialmente mediante la creación de espacios institucionales en el Ministerio de Educación y la producción de documentos de política, y luego mediante un juicioso ejercicio de difusión del nuevo proyecto. Así, en agosto de 1985 se realizó el Primer Seminario de Etnoeducación, convocado conjuntamente por el Ministerio de Educación y la Organización Nacional Indígena de Colombia (ONIC), con el patrocinio de la Interamerican Foundation. El documento de memorias incluye un apartado de conclusiones y recomendaciones (Colombia, MEN y ONIC I986, I35-I39), entre ellas la consolidación del equipo del Ministerio en el tema, la ampliación de la participación indígena en las decisiones sobre la política y diversos aspectos relativos a la situación educativa de algunos grupos indígenas a nivel local y regional, en un claro ejemplo de la manera en que se diseñan las técnicas de gobierno de los indígenas en el campo educativo, frecuentemente con el aval de las mismas poblaciones involucradas.

Como parte del proceso de difusión del proyecto, el Ministerio realizó un conjunto de seminarios regionales de etnoeducación, en los que participaron maestros, funcionarios, organizaciones indígenas y autoridades tradicionales. Entre I987 y I988 se realizaron talleres en Caquetá, Meta, Guaviare, Guainía y Amazonas, entre otros (Bodnar y Carrioni I987a, I987b, I988a, I988b, I988c). Para la misma época, el Ministerio publicó el libro Educación bilingüe: comunidad, escuela y currículo (Colombia, MEN s.f.), que era una adaptación de un texto publicado por la Unesco en Chile, en el que se ofrecía una guía de trabajo para maestros, que incluía orientaciones acerca de contenidos y metodologías que deberían emplearse en el trabajo en las aulas. Mientras tanto, algunas organizaciones continuaban su trabajo de diseño de materiales 
educativos, en ocasiones por su propia cuenta o con cooperación internacional, y en otras con el apoyo profesional y financiero del Ministerio. También se avanzó en la formación de maestros que trabajaban en escuelas de población indígena, proceso que dio lugar a los programas de profesionalización de docentes y a la posterior titulación de estos como bachilleres pedagógicos; más adelante estos programas servirían como referente para la creación de programas universitarios de etnoeducación (Caicedo y Castillo 2008). A lo largo de todo este proceso el Ministerio de Educación mantuvo una política bastante irregular en cuanto a la conformación de sus equipos de trabajo, aunque hubo un aspecto que fue objeto de constante requerimiento por parte de algunas organizaciones sociales: la contratación de activistas e intelectuales indígenas y negros para que se desempeñaran como funcionarios en el diseño y la ejecución de las políticas estatales; fue así como algunos intelectuales provenientes de procesos organizativos regionales o nacionales se posicionaron como expertos en etnoeducación. Valga mencionar que un número importante de ellos se desempeñan aún como funcionarios públicos en instituciones del sector educativo, y gestionan proyectos de cooperación internacional en etnoeducación, o como maestros en instituciones educativas locales, de modo que constituyen un importante sector de la intelectualidad étnica en el país.

Para comienzos de la década de los noventa, en apenas diez años, la etnoeducación abandonó el énfasis en la construcción de autonomía que se expresaba en las primeras formulaciones de la política, a la luz del concepto de etnodesarrollo, para concentrarse en la dimensión culturalista de conservación y recuperación de tradiciones, como puede observarse en las memorias del II Seminario de Etnoeducación sobre Diseño Curricular y I de Profesionalización, realizado en 1993 (Colombia, MEN 1998), en el que el énfasis en aspectos pedagógicos y de diseño de política pública también es notorio. El seminario tiene, además, implicaciones en otros planos, ya que es uno de los primeros documentos en que se registra participación de población afrocolombiana y se hace referencia a ella como un grupo étnico. Se menciona incluso que, dado el reciente reconocimiento de esta población como grupo étnico, aún es difícil para los participantes referirse a sí mismos en estos términos: 
Dicen que, a partir de la Constitución de I99I, son reconocidos como etnia; por lo tanto, no tienen unos fundamentos como tales; también expresan que hay una diferencia entre raizales y afrocolombianos, pero que tienen puntos comunes en algunos términos, lingüísticos. Se ha venido trabajando por fundamentos educativos en algunas comunidades de manera muy particular como en Palenque, Buenaventura, Chocó y otros pueblos afrocolombianos. (Colombia, MEN I998, 3I)

A los afrocolombianos y raizales les resultaba difícil expresarse en términos que se correspondieran con su nueva condición de grupo étnico, situación que ilustra una de las paradojas de la etnicidad: se supone que estos grupos son reconocidos como étnicos en tanto ya lo eran pero no se les incluía en tal categoría; no obstante, la etnicidad es una categoría académica, jurídica y política llena de contenidos específicos a la que ellos deberán amoldarse. Esta dificultad se observa en la mención que se hace de las experiencias educativas realizadas en comunidades negras hasta ese momento, cuyo lenguaje y objetivos eran distintos de los que para la fecha se habían institucionalizado entre los indígenas (Colombia, MEN I998, 3I). Lo mismo sucedió cuando, en 1993, el Ministerio de Educación realizó en Cartagena el Primer Seminario Taller de Etnoeducación para Comunidades Afrocolombianas (Colombia, MEN I994), en respuesta a uno de los compromisos fijados en el taller anterior; allí se presentaron como experiencias etnoeducativas diversos proyectos que se adelantaban desde mucho antes de que existiera tal denominación. Uno y otro caso ilustran la manera en que opera la violencia epistémica de estas políticas. El hecho de que a partir del cambio constitucional de 199I, y específicamente desde la promulgación de la Ley

IO El artículo I. ㅇ de la Ley 70 de 1993 reza: "La presente ley tiene por objeto reconocer a las comunidades negras que han venido ocupando tierras baldías en las zonas rurales ribereñas de los ríos de la Cuenca del Pacífico, de acuerdo con sus prácticas tradicionales de producción, el derecho a la propiedad colectiva, de conformidad con lo dispuesto en los artículos siguientes. Así mismo tiene como propósito establecer mecanismos para la protección de la identidad cultural y de los derechos de las comunidades negras de Colombia como grupo étnico, y el fomento de su desarrollo económico y social, con el fin de garantizar que estas comunidades obtengan condiciones reales de igualdad de oportunidades frente al resto de la sociedad colombiana". 70 de $1993^{10}$, se reconozca a las comunidades negras como grupo étnico, tiene efectos significativos tanto en el tratamiento que el Estado da a las poblaciones que empiezan a reclamar este estatus, como en la manera en que son concebidos los proyectos educativos que se venían ejecutando. En este contexto, la noción de 
grupo étnico, además de productora de alteridad, se basaba - $\mathrm{y}$ aún hoy lo sigue haciendo- en un molde indígena. Esto significa que no solo produce la idea de otredad, sino que lo hace a partir de un referente particular desde el cual se piensa la diferencia.

En 1996 el Ministerio presentó unos nuevos lineamientos de etnoeducación. En ese año la política de etnoeducación, que desde 1986 era concebida como política educativa indígena (Bodnar 1986), expresó de manera más clara un cambio formal, con el título de La etnoeducación. Realidad y esperanza de los pueblos indígenas y afrocolombianos (Colombia, MEN 1996a), y vino acompañada de un documento de Lineamientos generales para la educación en las comunidades afrocolombianas (Colombia, MEN I996b) ${ }^{11}$. No obstante, si bien la Ley 70 de 1993 había establecido la Cátedra de Estudios Afroco-

II Resulta por lo menos curioso que en la carátula del documento el título haga referencia a educación, mientras en su contenido se habla de etnoeducación. lombianos (artículo 39), esta no se reglamentó sino hasta 1998 (Decreto II22), y solo se publicó una primera propuesta en 200 I (Colombia, MEN 20OI); dilaciones que expresan las distintas lógicas
de pensamiento sobre la alteridad que han definido el lugar de la indianidad y la negridad en el país. Esta situación talvez ayude a entender por qué la interculturalidad ha ocupado un lugar marginal en los proyectos dirigidos a las poblaciones negras: su lugar en la producción académica acerca de lo cultural, en la legislación internacional y en las políticas de Estado ha sido distinto del lugar de quienes han sido la representación oficial de la otredad, los indígenas.

Desde el inicio de su institucionalización, en 1982, la difusión de la etnoeducación ha sido objeto de atención compartida por parte de indígenas y funcionarios estatales, al tiempo que se creó una amplia legislación relativa al tema, se produjeron documentos de orientación curricular y materiales didácticos y se abrieron programas de formación de maestros en al menos siete universidades. Aun así, y aunque con frecuencia han reclamado del Estado el cumplimiento de la legislación, las organizaciones sociales y algunos intelectuales indígenas y afrodescendientes han manifestado insistentemente su distancia frente a las políticas de Estado, y en muchos casos se han resistido a emplear el término etnoeducación para referirse a sus propios proyectos educativos (Bolaños et ál. 2004, 62). Al respecto, Abadio Green, 
uno de los intelectuales indígenas más destacados en el país, ha planteado que:

Los pueblos indígenas debimos aceptar y promover, incluso con una insistencia digna de mejores causas, la educación intercultural bilingüe егв o etnoeducación. Pero las razones para que ello ocurriera no fueron la aceptación de sus fundamentos y ni siquiera el reconocimiento de sus éxitos, sino su avance frente a la educación evangelizadora e integracionista que había. Obviamente ello condujo a que las propias organizaciones indígenas promovieran la concepción de etnoeducación y considero que otro tanto ocurrió con la ЕІB [...]. (Citado en Regalsky 2002)

Estas tensiones también se expresan en algunas conceptualizaciones elaboradas por el intelectual afrocolombiano Jorge García, para quien el proyecto educativo de las poblaciones negras y afrocolombianas se distancia de aquel que representa un proyecto de nacionalidad homogénea y los intereses de las élites nacionales:

El concepto de etnoeducación que se ha filtrado entre líneas del lenguaje institucional, revela la presencia de un pensamiento que busca la reafirmación de lo afro hacia dentro, en un intento por perfilar una propuesta educativa anclada en los intereses colectivos y no tanto en el interés ciudadano. Esta posibilidad de construir educación desde las raíces, en franca relación con los elementos de la identidad y fundamentalmente con el proyecto político-organizativo, es lo que llamamos etnoeducación en una perspectiva endógena. (García 2009, 28)

Como se puede observar, luego de casi tres décadas, ni los programas de etnoeducación ni las entidades y organizaciones que los promueven son homogéneos, ni lo son sus propósitos, ámbitos y estrategias de acción. Sin embargo, habría que analizar hasta dónde estas diferencias expresan una distancia real entre los proyectos etnoeducativos que son promovidos por el Estado y los que son propuestos por intelectuales con actitudes críticas respecto de los primeros. En concreto, habría que analizar cuán distintos son los proyectos políticos y las formas de entender la alteridad que constituyen a unos y otros, y habría que preguntarse si no constituyen dos expresiones de un mismo proyecto. 


\section{A MODO DE CIERRE: PEDAGOGíAS DE LA ALTERIDAD}

$n$ un contexto en que la cultura adquiere centralidad en tantos

y tan diversos frentes, hay un asunto que los atraviesa a todos: L la tensión entre unidad y diferencia, la relación entre mismidad y alteridad. Los debates académicos, las políticas estatales, los organismos multilaterales y las organizaciones sociales se refieren de alguna manera al problema de cómo afrontar el hecho de que en cada sociedad, y en las relaciones entre diferentes sociedades, existan trayectorias históricas y circunstancias presentes mediadas por relaciones desiguales de poder. La idea de cultura parece ocultar este hecho a fuerza de hacerlo visible; supone con bastante frecuencia que las diferencias son ontológicas, no construidas en relaciones históricas. Desconoce que la diferencia es relación, asume la otredad como substancia. Esta es una de las paradojas del reconocimiento multicultural: otorga derechos mientras ello no altere el orden establecido.

\section{Pedagogías de la alteridad}

a etnoeducación no es solo una política pública de educación para grupos étnicos, ha sido también una política de producción y de -gobierno de la alteridad. Desde sus inicios ha estado ligada a la problematización de las relaciones entre grupos culturales y ha sido espacio de conceptualización de la multiculturalidad y de definición de los mecanismos para administrarla. La etnoeducación no solo es un derecho de los grupos étnicos, entre otras razones porque ella define quiénes son los grupos étnicos, cómo deben educarse y el tipo de relación que deben establecer con los conocimientos y poblaciones que son considerados como no étnicos. Sus currículos están diseñados de tal forma que los sujetos que en ella se educan actúen de acuerdo con una condición de radical alteridad respecto de las sociedades de las que hacen parte; es decir, para que se gobiernen a sí mismos en nombre de la cultura y la diferencia.

Tanto en la academia como en las instituciones del Estado y las organizaciones sociales, la etnoeducación es concebida y demandada como educación de los grupos étnicos y para ellos: 
busca diseñar y poner en práctica programas de educación que fortalezcan las identidades y culturas de estos grupos. Como proyecto pedagógico, promueve una formación centrada en un conjunto de aspectos definidos principalmente por la antropología - y por otras disciplinas, como la lingüística-, que se supone encarnan las culturas de indígenas y afrodescendientes ${ }^{12}$; en la mayoría de los casos se trata de atributos escasamente presentes en la vida cotidiana de los grupos o en su conocimiento local, por lo que resulta común que se recurra a nociones como las de recuperación y fortalecimiento cultural. En casi todos los casos, aquellos rasgos que se consideran como propios están definidos por una idea particular de cultura, marcada por lo que se podría llamar el deber de la diferencia cultural. Los grupos étnicos están en la obligación de hacer visible, recuperar, y fortalecer su cultura, a la que se presenta como inconmensurable y siempre en aparente oposición con la que se considera la cultura dominante, moderna, occidental, eurocéntrica o universal; es decir, una cultura subalterna, tradicional, no occidental, no eurocéntrica y local. Como todo proyecto pedagógico, la etnoeducación busca formar un tipo particular de sujetos, en este caso, sujetos étnicos. Es una pedagogía para la producción de alteridades, un proyecto educativo de otrerización.

Una característica frecuente de los proyectos de etnoeducación es la denuncia de los procesos de sometimiento e imposición cultural; sin embargo, la mayoría de estos proyectos carecen de un análisis crítico acerca de los efectos de esta historia, y tampoco mencionan las formas concretas en que se expresa en el presente $^{13}$. En consecuencia, es posible encontrar que se reclamen como propias instituciones coloniales, como el uso de castigos físicos para ejercer justicia (cepo, fuete), el cabildo como forma de gobierno, las unidades de medida de origen colonial (castellano, ración) o las fiestas

I2 Es frecuente encontrar referencias a la ancestralidad, las autoridades tradicionales, la lengua indígena, la existencia de mitos y leyendas, la medicina tradicional, las formas tradicionales de producción y la relación armónica con el ambiente, entre las más comunes.

I3 Talvez la mayor excepción se encuentra en los aspectos lingüísticos, en los que se hace hincapié en la pérdida de la lengua y en el impacto de la imposición del castellano. religiosas católicas (fiestas dedicadas a los patronos), entre otras. De esta manera, al ser incorporadas en los proyectos educativos, estas particulares formas de entender la historia promueven la idea de un sujeto esencialmente definido por su exterioridad con respecto a la formación social de la que participa. Es decir, 
proyectan la idea de grupos que constituyeron sus instituciones políticas, definieron sus prácticas jurídicas, adoptaron prácticas religiosas o llegaron a ser hablantes de un idioma por fuera de las relaciones sociales. Esto resulta bastante problemático, sobre todo si se quiere cuestionar la historia del colonialismo.

En este contexto, la interculturalidad se ocupa de hacer conmensurable la alteridad, en un proyecto ambiguo que, al tiempo que produce la alteridad, busca integrarla. Opera como fin o principio en nombre del cual se busca evitar que aquellos sujetos de la otredad permanezcan al margen del proyecto de nación: gestiona un particular diagrama de mismidades y diferencias, para el que traza unos contornos bien definidos. Al promover el diálogo entre culturas, propende por que los otros no aprendan solo el conocimiento propio, sino que aprendan también el universal. Dado que es un proyecto dirigido únicamente a quienes se reconozcan y sean reconocidos como sujetos étnicos, más que de interculturalidad se trata de intraculturalidad: solamente afecta a aquellos que se gobiernan en nombre de la cultura.

La interculturalidad opera como principio de ordenamiento de lo social, marcando, clasificando, incluyendo y excluyendo expresiones de la diferencia que ella misma produce. No se trata pues de que las diferencias culturales estén allí y de que los proyectos interculturales sean una forma de llegar a tramitar las relaciones entre ellas; de hecho, muchas diferencias culturales no son objeto de ningún tipo de atención intercultural. Estos proyectos intervienen sobre producciones específicas de la diferencia, en particular, sobre aquellas en las que la diferencia opera como alteridad y es clasificada como cultural. No se trata entonces de problematizar cualquier tipo de diferencia, sino un tipo particular en nombre del cual se argumenta la supuesta necesidad de intervenir y transformar las relaciones entre grupos humanos. A ello hay que sumar que la problematización de las relaciones entre culturas no produce los mismos efectos para los distintos polos de la relación. Las poblaciones "mayoritarias" son con frecuencia ignoradas en los programas de gobierno intercultural. De tal manera, se produce primero un régimen particular desde el cual se piensa la diferencia y se problematiza la historia, o la situación actual de su relación con los sectores de la sociedad que encarnan el proyecto de mismidad. Desde este proyecto se enuncian las diferencias, y desde ese lugar se interviene sobre el polo ubicado como alteridad en la relación. 
La etnoeducación llegó a ser posible en un momento en el que la cultura adquiría su centralidad y se dispersaba de manera insospechada en los más diversos ámbitos académicos y políticos, como producto de un proceso histórico en el que se cruzaron múltiples líneas de fuerza, entre ellas, la problematización de la existencia de grupos étnicos dentro de las sociedades nacionales; los cuestionamientos a la noción de raza, que adquirieron su fuerza decisiva luego de la segunda guerra mundial y que se relacionan estrechamente con la institucionalización de la antropología y los debates sobre el racismo en los Estados Unidos (Grimson 2008; Trouillot 20II); los debates propuestos por movimientos intelectuales como el de la negritud, que resultarían esenciales en las luchas anticoloniales y en la transformación de los imaginarios coloniales de la negridad (Césaire 2006); la constitución de un sistema interestatal de derecho internacional, que incluyó los derechos humanos y los derechos sociales y culturales; la creación de instancias para la lucha en contra de las diferentes formas de discriminación y racismo (Anaya 2005); los cuestionamientos al colonialismo, particularmente en la segunda posguerra, que resultaron cruciales en discusiones como las de la autodeterminación de los pueblos y en la emergencia de los "indígenas" como sujetos de derecho (Anaya 2005; Morales 200I) ${ }^{14}$; la consolidación de un sistema institucional interestatal orientado

I4 En este contexto, la noción de indígena aparece para nombrar a los pueblos nativos de aquellos territorios que padecieron la colonización.

a la integración de los indígenas a las sociedades nacionales y al desarrollo (Ballesteros y Ulloa I96r; Unesco 1996); y la creación y consolidación de organizaciones sociales indígenas (Bonfil I979; Gros 2000), entre las líneas más visibles.

Es así que las luchas y proyectos contemporáneos marcados por la diferencia cultural y la etnicidad no pueden ser entendidos como mera resistencia u oposición a un poder por fuera del cual se encontrarían. Al contrario, los términos de las luchas son definidos de acuerdo con las formas de poder propias del momento histórico. Más que como un término o un concepto en sí mismo, la interculturalidad resulta relevante como proyecto político que participa de la producción y gestión de la alteridad; un proyecto que debe ser comprendido en su complejidad, si es que se quiere hacerle resistencia.

Los planteamientos que afirman que la etnoeducación es un derecho étnico por el que se debe luchar, en tanto expresa 
las aspiraciones y proyectos políticos de indígenas y afrodescendientes, o aquellos que sostienen que la interculturalidad es un proyecto político y epistémico alternativo nacido en las organizaciones sociales, son, en el mejor de los casos, ingenuos: desconocen la complejidad de los procesos en que se inserta la experiencia contemporánea del multiculturalismo. Si se quiere aportar a la construcción de proyectos políticos alternativos con capacidad real para transformar las relaciones de poder vigentes, es necesario develar cómo opera ese poder en formaciones sociales concretas y en momentos particulares. Para ello es crucial comenzar por comprender las formas en que la diferencia es articulada a formas de gobierno que sostienen, reproducen y legitiman la desigualdad, sobre todo en aquellos casos en que esto implica ir en contravía de las certezas propias del sentido común de la época.

\section{REFERENCIAS}

Aguirre Beltrán, GonZalo. 1957. El proceso de aculturación. México, D. F.: Universidad Nacional Autónoma de México.

ANAYA, S. JAMEs. 2005. Los pueblos indígenas en el derecho internacional. Madrid: Trotta.

Arana de Swadesh, Evangelina. 1976. "Lingüística y educación indígena”. En América Indígena xxxvi (2): 347-358.

Bairon, Max Agatón. 1952. "La educación indígena en las selvas de Bolivia”. América Indígena XII (2): I4I-I47.

Ballesteros Gaibrois, Manuel y Julia Ulloa Suárez. ig6i. Indigenismo americano. Madrid: Cultura Hispánica.

Bodnar, Yolanda. 1986. Etnoeducación. Política educativa indígena nacional y algunas experiencias bilingües-interculturales adelantadas en el país. Bogotá: Ministerio de Educación Nacional.

Bodnar, Yolanda y Gina Carrioni. 1987a. Informe Seminario de Etnoeducación Mama Bwe Reojache - Caquetá. Agosto 24-28. Bogotá: Ministerio de Educación Nacional, Dirección General de Capacitación y Perfeccionamiento Docente, Currículo y Medios Educativos, Programa de Etnoeducación.

Bodnar, Yolanda y Gina CarRIOni. 1987b. Informe Seminario de Etnoeducación Santa Teresita - Vichada, del 5 al io de julio y Seminario para 
la Unificación del Material Educativo de la Comunidad Sikuani de Meta y Vichada. El Cortijo (Meta), agosto 3 I a 4 de septiembre. Bogotá: Ministerio de Educación Nacional, Dirección General de Capacitación y Perfeccionamiento Docente, Currículo y Medios Educativos, Programa de Etnoeducación.

Bodnar, Yolanda y Gina Carrioni. I988a. Informe Seminario de Etnoeducación. Puerto Inírida - Guainía. Noviembre 2 al 6 de 1987. Bogotá: Ministerio de Educación Nacional, Dirección General de Capacitación y Perfeccionamiento Docente, Currículo y Medios Educativos, Programa de Etnoeducación.

Bodnar, Yolanda y Gina Carrioni. I988b. Informe Seminario de Etnoeducación. Leticia, Amazonas. Noviembre 30 a diciembre 4. Bogotá: Ministerio de Educación Nacional, Dirección General de Capacitación y Perfeccionamiento Docente, Currículo y Medios Educativos, Programa de Etnoeducación.

Bodnar, Yolanda y Gina Carrioni. I988c. Seminario para la Elaboración de Material Educativo para la Etnia Sikuani y de una Propuesta de Programa de Profesionalización para Maestros Bilingües Sikuani. Centro Unuma - Puerto Gaitán (Meta). 22 a 24 de abril de i988. Bogotá: Ministerio de Educación Nacional, Dirección General de Capacitación y Perfeccionamiento Docente, Currículo y Medios Educativos, Programa de Etnoeducación.

Bolaños, Graciela, Carlos Miñana, Abelardo Ramos y Joanne Rappaport. 2004. ¿Qué pasaría si la escuela...? 30 años de construcción de una educación propia. Bogotá: Programa de Educación Bilingüe e Intercultural, Consejo Regional Indígena del Cauca.

Bonfil BATAlla, GuILlERMO. I979. "Las nuevas organizaciones indígenas. (Hipótesis para la formulación de un modelo analítico)”. En Indianidad y descolonización en América Latina. Documentos de la Segunda Reunión de Barbados, editado por Grupo de Barbados, 23-40. México, D. F.: Nueva Imagen.

BONFIl BATAlla, GuILLRmo. I982. "El etnodesarrollo: sus premisas jurídicas, políticas y de organización”. En América Latina: etnodesarrollo y etnocidio, editado por Francisco Rojas Aravena, I3I-I45. San José: Flacso.

Bonilla, Víctor Daniel, Gonzalo Castillo, Orlando Fals Borda y Augusto LiBreros. I972. Causa popular, ciencia popular. Una metodología del conocimiento científico a través de la acción. Bogotá: Publicaciones de la Rosca.

Caicedo, José Antonio y Elizabeth Castillo. 2008. "Indígenas y afrodescendientes en la universidad colombiana: nuevos sujetos, viejas estructuras”. Cuadernos Interculturales 6 (Iо): 62-90. 
CÉSAIre, Aimé. 2006. Discurso sobre el colonialismo. Madrid: Akal.

Colombia, Ministerio de Educación Nacional (men). i994. Primer Seminario Taller de Etnoeducación para Comunidades Afrocolombianas. Memorias. Bogotá: MEN.

Colombia, MEN. I996a. Etnoeducación: realidad y esperanza de los pueblos indígenas afrocolombianos. Bogotá: MEN.

Colombia, mEn. I996b. Lineamientos generales para la educación en las comunidades afrocolombianas. Bogotá: MEN.

Colombia, men. I998. II Seminario de Etnoeducación sobre Diseño Curricular y I de Profesionalización. El Ocaso, I7 al 28 de mayo de 1993. Memorias. Bogotá: MEn, Dirección General de Investigación y Desarrollo Pedagógico, Grupo de Atención a la Comunidad Educativa y Grupos Poblacionales, Programa Nacional de Etnoeducación.

Colombia, MEN. 200I. Cátedra de Estudios Afrocolombianos. Lineamientos curriculares. Bogotá: MEN.

Colombia, MEN. s. f. Educación bilingüe: comunidad, escuela y currículo. Plan de Universalización de la Educación Primaria. Materiales de apoyo para la formación docente en educación bilingüe intercultural CULTURA. Bogotá: MEN, Dirección General de Capacitación, Programa de Etnoeducación.

Colombia, MEn y Organización Indígena de Colombia (ONIC). I986. Primer Seminario de Etnoeducación. 25-3I de agosto de ig85. Memorias. Bogotá: MEN y ONIC.

Colombia, Ministerio de Gobierno. I972. Primera Reunión de Trabajo sobre Educación Binlingüe en los Grupos Indígenas. Documento final. Bogotá: Dirección General de Integración y desarrollo de la Comunidad, Ministerio de Gobierno.

Comité de Educación Bilingüe Aborigen Sikuani (cebas). ig88. Wajajume Itane Yakinatsi Yorobatsi. Bogotá: Cebas.

Cuche, Denys. I966/2007. La noción de cultura en las ciencias sociales. Buenos Aires: Nueva Visión.

Cunin, Elisabeth. 20I2. “¿Es posible hablar de 'diáspora negra’ en América latina? Etnicidad, nación y globalización a partir del caso colombiano”. En prensa. Una versión en francés fue publicada en Autrepart 38: I35-I53, 2006.

Díaz-Polanco, Héctor, Francisco Javier Guerrero, Víctor Bravo, Leopoldo Allub, Marco A. Michel y Lourdes ArizPe. I979/1987. Indigenismo, modernización y marginalidad. Una revisión crítica. México, D. F.: Centro de Investigación para la Integración Social.

Escobar, Arturo. 20io. Territorios de diferencia. Lugar, movimientos, vida, redes. Popayán: Envión. 
Fals Borda, Orlando. I985. Conocimiento y poder popular. Lecciones con campesinos de Nicaragua, México, Colombia. Bogotá: Punta de Lanza y Siglo XXI.

Foucault, Michel. 2006. Seguridad, territorio, población. Buenos Aires: Fondo de Cultura Económica.

Freire, Paulo. 200o. Pedagogía del oprimido. México, D. F.: Siglo XXI.

García, Jorge. 2009. Sube la marea. Educación propia y autonomía en los territorios negros del Pacífico. Tumaco: Edinar.

Giroux, Henry. 2004. Teoría y resistencia en educación. México, D. F.: Siglo XXI.

Grimson, AlejANDRO. 2008. "Diversidad y cultura. Reificación y situacionalidad”. Tabula Rasa 8 (enero-junio): 45-67.

Gros, Christian. 200o. Políticas de la etnicidad: identidad, Estado y modernidad. Bogotá: Instituto Colombiano de Antropología e Historia.

INDA, JONATHAN XAVIER. 2008. "Analytics of the Modern: An Introduction”. En Anthropologies of Modernity, editado por Jonathan Xavier Inda, I-20. Oxford: Blackwell.

INSTITUTO INTERAMERICANO INDIGENISTA (III). I968. “VI Congreso Indigenista Interamericano: ponencias”. Anuario Indigenista XXVIII (diciembre).

Marroquín, Alejandro Dagoberto. I972. Balance del indigenismo: informe sobre la política indigenista en América. México: Instituto Indigenista Interamericano.

Montalto, Nicholas. 1978. The Forgotten Dream: A History of the Intercultural Education Movement, I924-I94I. Minneapolis: University of Minnesota.

Montoya, JAviER. I972. "Reportaje a un profesor indígena bilingüe”. Cultura Nariñense 53 (noviembre): 27-29.

Morales, Patricia (coord.). 200I. Pueblos indígenas, derechos humanos e interdependencia global. México, D. F.: Siglo XXI.

Mosquera, JuAn De Dios. 1986. Las comunidades negras de Colombia. Pasado, presente y futuro. Medellín: Movimiento Cimarrón.

Nahmad Sitton, Salomón. I982. "La educación bilingüe y bicultural para las regiones interculturales de México”. América Indígena XLII (2).

Paulston, Rolland G. I970. "Maestros como agentes del cambio comunal: cuatro programas peruanos”. América Indígena XXX (4): 929-944.

RAPPAPORT, JOANNE. 2008. Utopías interculturales. Intelectuales públicos, experimentos con la cultura y el pluralismo étnico en Colombia. Bogotá: Editorial Universidad del Rosario, Universidad del Cauca. 
RedDen, John y Francis A. Ryan. I95I. Intercultural Education. Milwaukee: Bruce Publishing.

Regalsky, Pablo. 2002. "Reforma educativa y territorialidad andina en Bolivia: en busca de los protagonistas”. En Interculturalidad y política: desafíos y posibilidades, editado por Norma Fuller, I43-I63. Lima: Red para el Desarrollo de las Ciencias Sociales en el Perú.

Rubio, Gonzalo. 1957. Promociones indígenas en América. Quito: Casa de la Cultura Ecuatoriana.

SANTAMARÍA, Ángela. 2008. Redes transnacionales y emergencia de la diplomacia indígena. Bogotá: Centro de Estudios Políticos e Internacionales, Universidad del Rosario.

Sosa, Marcelino. I98I. El niño guahibo y la educación bilingüe. Bogotá: Marcelino Sosa.

Torres, Rosa María. I986. Educación popular: un encuentro con Paulo Freire. Caracas: Cooperativa Laboratorio Educativo.

Trouillot, Michel-Rolph. 20II. Transformaciones globales. La antropología y el mundo moderno. Popayán: Universidad del Cauca y Universidad de los Andes.

Organización dE LAS NACiONES UnidAs PARA LA EDUCACión, LA CiENCIA Y LA Cultura (Unesco). I996. Nuestra diversidad creativa: Comisión Mundial de Cultura y Desarrollo. México, D. F.: Unesco.

Unesco y Facultad Latinoamericana de Ciencias Sociales (Flacso). I98I. Etnocidio y etnodesarrollo. Ginebra: Docip.

VArgas, Nicasio A. y Jacob A. LoEwEn. I963. "Experiencia de alfabetización con los indios chocoes”. América Indígena XXIII (2): I2I-I25.

WAde, Peter. 2007. "Defining Blackness in Colombia”. Ponencia presentada en el XII Congreso de Antropología en Colombia. Universidad Nacional de Colombia, Bogotá.

Walsh, Catherine. 2004. "Geopolíticas del conocimiento, interculturalidad y descolonialización”. Boletín ICCI-ARY Rimay 6 (60). Recuperado el 30 de octubre de 20II, http://icci.nativeweb.org

Walsh, Catherine. 2009. Interculturalidad, Estado, sociedad. Luchas (de)coloniales de nuestra época. Quito: Universidad Andina Simón Bolívar y Abya Yala.

Walsh, John E. I973. Intercultural Education in the Community of Man. Honolulu: The University Press of Hawaii. 